\title{
AN IMAGE PROCESSING ARCHITECTURE TO ENHANCE IMAGE CONTRAST
}

\author{
Yue-Der LiN ${ }^{1}$, Hen-Wei TSAO ${ }^{2}$ AND FoK-CHING CHONG ${ }^{2}$ \\ ${ }^{1}$ School of Post Baccalaureate Chinese Medicine, China Medical College, Taichung \\ ${ }^{2}$ Institute of Electrical Engineering, National Taiwan University, Taipei, Taiwan
}

\section{ABSTRACT}

To have a good image contrast is an important issue in medical images. This paper introduces a feedback-type image processing architecture that can enhance image contrast without further digital image processing technique, e.g. histogram equalization. Compared with the conventional open-loop imaging system, the images derived by the proposed method has a full-range histogram without causing image distortion, and this is difficult to attain for open-loop imaging system.

Biomed Eng Appl Basis Comm, 2002 (October); 14: 215-217.

Keywords: image contrast, feedback, image processing, histogram equalization.

\section{INTRODUCTION}

Good image contrast can give better effect of vision, it is especially important for medical image where the slight anatomic or physiological feature may lead to different diagnostic result. The image contrast can be estimated by the range of gray level values occupied by the pixels in the histogram. It is usually not easy to acquire a digital image with full-range gray level using commercial products currently available. The general approach to serve the purpose in digital image processing is the histogram-modification technique. However, such an approach usually results in image distortion [1]. In this study, we propose a feedback-type image processing architecture to acquire the images with full-range gray level. Only two digital-toanalog converters (DAC) and some logic components were added as compared with the commercial product. The proposed architecture has the advantages of no

Received: August 20, 2002; Accepted: Oct. 18, 2002 Correspondence: Yue-Der Lin,

School of Post Baccalaureate Chinese Medicine China Medical College,

91, Hsueh-Shih Road, North District, Taichung 404, Taiwan

Email: ydlin@mail.cmc.edu.tw image distortion, easy implementation, and low cost.

\section{ARCHITECTURE DESCRIPTION}

The block diagram of the proposed architecture is demonstrated in Figure 1. The timing control circuit is not depicted in this figure for conciseness. The main feature is that the reference voltages of the analog-todigital converter (ADC) can be adjusted automatically during the process of image acquisition. The reference voltages are controlled via the DACs. The procedure of image acquisition is shown in Figure 2. From this flowchart, two samplings are experienced for each acquired image. For the first sampling, the maximum and the minimum gray levels of the pixels are determined and fed back to calibrate the reference voltages of $A D C$ via two DACs. And thus the frame grabbed at the second time can have a full-range histogram.

Some limits should be noted for the proposed architecture. Firstly, the environmental light sources should not change during image acquisition. And, because each images experiences two samplings, the proposed architecture is not recommended for dynamic image acquisition due to the time interval between two successive samplings. Besides, the digitized resolution should be limited for the sake of the unavoidable intrinsic noise of image sensor [2]. Let $S N R$ represent the signal-to-noise ratio (SNR) of the image sensor in decibel $(\mathrm{dB})$, and an $N$-bit ADC is used. Then the 




Fig. 1 The block diagram of the proposed architecture.



Fig. 2 The image acquisition procedure for the proposed system.

following condition must be satisfied to avoid image distortion.

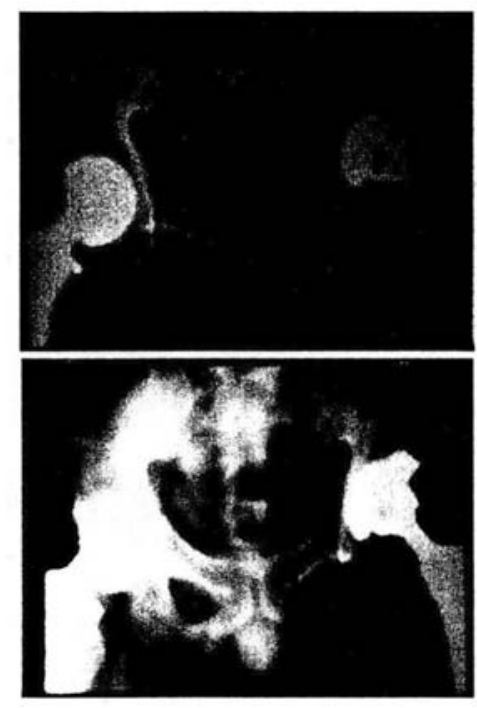

Fig. 3 The results of image grabbed by the commercial product (upper) and by the proposed system (lower).

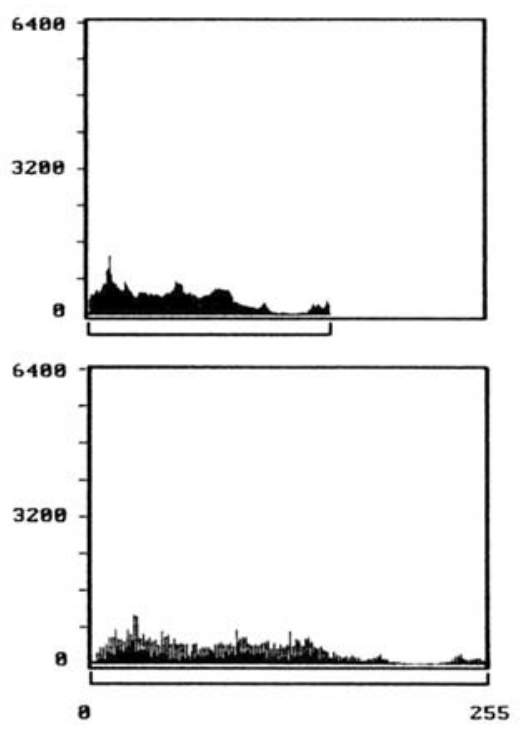

Fig. 4 The histogram of the images in Figure 3 correspondingly, where $x$-axis indicates the pixel values and $y$-axis represents the number for each corresponding pixel value.

$$
S N R>20 \log 2^{N}
$$

For example, if the $S N R$ of the image sensor is 50 $\mathrm{dB}$, an 8-bit ADC can be used for image digitization.

\section{RESULT}

A PC-based imaging system $(256 \times 256 \times 8$, black 
and white) based on the architecture depicted in Figure 1 was designed to verify the feasibility of the proposed architecture. The image sensor is a standard CCD camera with 50-dB SNR. The images grabbed by our system were compared with the same image grabbed by a commercial product (VFG Frame Grabber, Visionetics Corp.) at the same environmental conditions. The performance evaluation results for an X-ray image are given in Figure 3 and Figure 4. In Figure 3, the upper image is derived from the commercial product (VFG Frame Grabber, Visionetics Corp.) and the lower image is acquired from our system. The corresponding histograms are demonstrated in Figure 4. From the results, the proposed system proves to be able to enhance the image contrast without causing distortion.

\section{DISCUSSION \& CONCLUSION}

An image processing architecture to enhance the contrast of static image without causing distortion is proposed in this study. Compared with a typical commercial frame grabber system (VFG Frame Grabber, Visionetics Corp.), the proposed method proves to be feasible for acquiring a static image with enhanced contrast. Although the medical images are used for verifying its performance, the proposed method is also recommended for general-purpose image processing.

\section{REFERENCE}

1. Gonzalez RC and Wintz P: Digital Image Processing, 2nd Ed., (Addison-Wesley, Massachusetts) 1987: 144-160.

2. Motchenbacher $\mathrm{CD}$ and Connelly JA: Low-Noise Electronic System Design, (John Wiley \& Sons, New York) 1993: 188-194. 